\title{
Relação entre autoestima, nível intelectual geral e metacognição em adolescentes
}

\author{
Luma Tiziotto Deffendi \\ Universidade Federal de São Carlos - SP \\ Patrícia Waltz Schelini \\ Universidade Federal de São Carlos - SP
}

\begin{abstract}
Resumo
O objetivo do presente estudo constitui-se em verificar se o conhecimento de jovens sobre suas capacidades cognitivas, o chamado conhecimento metacognitivo, corresponde ao desempenho real em tarefas cognitivas e ao nível em que a autoestima está envolvida nessa relação. Participaram do estudo 61 alunos, de ambos os sexos, de duas turmas de terceiro ano do Ensino Médio de uma escola particular do Interior do Estado de São Paulo, com idades variando entre 16 e 18 anos. Foram utilizados três instrumentos psicológicos: as Matrizes Progressivas de Raven; a Escala de Metacognição (EMETA) e a Escala de Autoestima de Rosemberg. Os escores obtidos nas três medidas foram correlacionados e os resultados indicaram a existência de uma correlação forte entre os escores da Escala de Metacognição e Autoestima, confirmando uma das hipóteses iniciais de que os componentes metacognitivos podem estar relacionados ao conceito de autoestima.
\end{abstract}

Palavras-chave: Autoestima, metacognição, inteligência.

\section{Self-esteem, overall intellectual level and metacognitive knowledge in teenagers}

\begin{abstract}
The purpose of this study is to investigate whether the knowledge teenagers have about their cognitive abilities, the metacognitive knowledge, measures up to their real performance in some cognitive tasks and in which level it is related to their self-esteem. Sixty-one students, both genders, ages between 16 and 18 years, from two differents senior classes of a private school in São Paulo state, participated in this study. They were submitted to three different psychological tests: the Raven's Progressive Matrices test, the Metacognition Scale (EMETA) and the Rosemberg Self-esteem Test. The scores on the three measures were correlated, and the results indicate the existence of a strong correlation between the scores of the Metacognition Scale and the Self-esteem Test. These results confirm one of the initial hypothesis that some of the participants' metacognitive components could be related to their self-esteem levels.
\end{abstract}

Key-words: Self esteem, metacognition, intelligence.

\section{Relación entre la autoestima, el nivel intelectual general y la metacognición en adolescentes}

\section{Resumen}

El objetivo de este estudio fue verificar si el conocimiento de jóvenes sobre sus capacidades cognitivas, Ilamado conocimiento metacognitivo, corresponde al rendimiento real en tareas cognitivas, y en qué nivel la autoestima está involucrada en esa relación. Participaron del estudio 61 alumnos de ambos sexos, de dos clases del tercer año de la Enseñanza Media de una escuela particular del Interior del Estado de São Paulo, con edades variando entre 16 y 18 años. Se utilizaron tres instrumentos psicológicos: las Matrizes Progresivas de Raven; la Escala de Metacognición (EMETA) y la Escala de Autoestima de Rosemberg. Los puntajes obtenidos en los tres instrumentos fueron correlacionados y los resultados señalaron la existencia de una fuerte correlación entre los puntajes de la Escala de Metacognición y Autoestima, confirmando una de las hipótesis iniciales de que los componentes metacognitivos pueden estar relacionados al concepto de autoestima.

Palabras Clave: autoestima; metacognición; inteligencia. 


\section{Introdução}

O estudo da inteligência se confunde com a própria história da Psicologia, uma vez que as primeiras pesquisas empíricas feitas neste campo tratavam de sua medida (Mendoza, \& Colom, 2006). A inteligência parece estar relacionada a muitas das nossas realizações do dia a dia, mais até do que poderíamos supor. É um dos atributos psicológicos mais valorizados socialmente, tendo assumido um valor fundamental ao longo da existência do homem, particularmente no que se refere à sua adaptação e sobrevivência (Faria, Pepi, \& Alesi, 2004). Há inúmeras definições e correntes teóricas que buscam facilitar sua compreensão. Howard (1993) considera que três conceitos estão mais frequentemente relacionados ao construto inteligência: 1- a inteligência como um fator geral (fator $g$ ), conceito que é assumido de um modo mais biológico e matemático; 2- como propriedade do comportamento adaptativo, no qual a inteligência pode ser mais um adjetivo que um substantivo, mais uma designação do que uma propriedade; 3- como conjunto de aptidões, sendo que para alguns estudiosos tais aptidões estão conectadas ao conhecimento, e para outros, não.

Segundo Sternberg (1981), podem-se considerar três grandes abordagens no estudo da inteligência: abordagem fatorial (diferencial ou psicométrica), abordagem desenvolvimentalista (evolutiva) e abordagem cognitivista ou do processamento da informação. Embora essas três abordagens (psicométrica, desenvolvimentalista e cognitiva) possuam fundamentações teóricas diferentes, todas elas procuram identificar elementos universais para a compreensão do comportamento intelectual. Dentre as correntes, o presente estudo será voltado à psicométrica, que entende a inteligência por meio de fatores.

Uma questão que permeia os teóricos da psicometria diz respeito à existência ou não de uma inteligência geral, o chamado fator $g$ (Santos, Noronha, \& Sisto, 2005). Em 1927 Spearman defendeu a ideia de que um fator geral, ou fator $g$, inicialmente concebido como uma energia mental inata, seria o responsável por explicar a correlação positiva entre dois testes de capacidade mental. Fatores específicos, ou fatores $s$, estariam envolvidos apenas em testes que dependam de aprendizagem. $O$ fator $g$ é um construto psicométrico e psicológico que descreve um conjunto de fenômenos que estão associados com os resultados do funcionamento mental humano. É uma ideia abstrata, latente. A inteligência, quando associada ao fator $g$, é entendida como um componente herdável e bastante estável, apesar de seu declínio na senilidade (Sisto, Ferreira, \& Matos, 2006).

Segundo Bernstein (1961), as melhores medições de $g$ são conseguidas por meio de testes homogêneos "de raciocínio matemático ou gramatical (de sinônimos, de oposição) ou de percepção de relações complexas com material visual e, em especial, os de material não verbal, com base em problemas de edução de relações" (p. 17). Diversas evidências apontam também a possibilidade de se avaliar o fator $g$ com a utilização de qualquer bateria de testes de inteligência (Primi, 2003), mesmo que alguns testes psico- lógicos, como as Matrizes Progressivas de Raven, possam ser considerados como boas ou melhores medidas do fator $g$ (Almeida, 1994).

\section{Da Inteligência/cognição à metacognição}

Segundo Brown (1987), é muito difícil precisar exatamente o que é meta e o que é da ordem da cognição. Sobre a cognição, aliás, vale dizer que, considerando-se toda a dimensão de teorias intelectuais, a inteligência não tende a ser vista como um construto diferente. Anderson (2004) afirmou que psicólogos e cientistas cognitivos usam a palavra "inteligência" para tratar da propriedade de todo o sistema cognitivo humano, de forma que "cognição" seria sinônimo de "inteligência". A compreensão que as pessoas têm de seu próprio processamento cognitivo é denominada pela Psicologia Cognitiva de metacognição. É através dela que podemos planejar nossas ações e corrigi-las quando estas não ocorrem como esperamos, ou ser capazes de monitorar nossos comportamentos e adequá-los a cada exigência. Pode-se entender a metacognição como o conhecimento e controle que a pessoa tem sobre sua própria cognição e atividades de aprendizagem. Isso implica em ter conhecimento do seu estilo de pensamento (processos e eventos cognitivos), de seu conteúdo (estruturas) e da habilidade para controlar esses processos, com o objetivo de organizá-los, revisá-los e modificá-los em função dos resultados obtidos na aprendizagem (Bolívar, 2002).

Flavell $(1976,1979)$ foi um dos precursores dos estudos sobre a metacognição, definindo-a como o "conhecimento e cognição sobre o fenômeno cognitivo" (Flavell, 1979, p.906), referindo-se ao conhecimento que uma pessoa tem sobre os próprios processos e produtos cognitivos. Com o intuito de especificar os domínios da metacognição, Flavell (1979) propôs o Modelo de Monitoramento Cognitivo (Model of Cognitive Monitoring). Neste modelo, o autor sugere que o monitoramento dos processos cognitivos ocorre por meio de ações e interações entre quatro classes de fenômenos: o conhecimento metacognitivo, as experiências metacognitivas, os objetivos cognitivos e as ações ou estratégias cognitivas.

Para elucidar o conceito de conhecimento metacognitivo de forma clara e objetiva, vale recorrer a Dunlosky e Metcalfe (2009), os quais afirmam que o conhecimento metacognitivo inclui todo o conhecimento adquirido relacionado a fatos, crenças e episódios que a pessoa pode verbalizar. Além deles, Ribeiro (2003) enfatiza que o conhecimento metacognitivo contribui nas tomadas de decisão, ajudando a identificar e representar as situações e assim facilitando o acesso às estratégias disponíveis, e também na escolha das que podem ser aplicadas, além de permitir avaliar os resultados finais ou intermediários a fim de continuar com a estratégia escolhida ou mudá-la em decorrência da avaliação realizada. No que se refere ao termo "controle", ele pode ser associado aos mecanismos de autorregulação, utilizados para coordenar e controlar ponderadamente as tentativas de aprender e resolver tarefas. Exemplos de atividades 
metacognitivas regulatórias são o planejamento das ações, ajustes das estratégias a serem utilizadas para alcançar um objetivo específico, o monitoramento das ações adotadas e a avaliação das estratégias.

As experiências metacognitivas referem-se à consciência das experiências cognitivas e afetivas que acompanham cada episódio cognitivo, podendo exercer grande influência sobre a atividade cognitiva, porque comumente estão relacionadas à percepção do sucesso (Boruchovitch, Schelini, \& Santos, 2010; Dunlosky, \& Metcalfe, 2009). Ribeiro (2003) enfatiza a importância das experiências metacognitivas, pois com elas o sujeito pode avaliar as suas dificuldades e traçar meios que possam ajudá-lo a dar uma solução a tais dificuldades. Para Figueira (1994), a experiência metacognitiva assume um papel importante na condução da vida intelectual do sujeito, pois é nela que o sujeito se baseia para saber em que ponto se encontra na atividade que está desenvolvendo, o progresso que já realizou e como está sendo o seu desempenho. A partir destas considerações, pode-se afirmar que as experiências metacognitivas e o conhecimento metacognitivo estão relacionados, pois, se por um lado o conhecimento permite interpretar as experiências e agir sobre elas, por outro, as ideias e sentimentos poderão contribuir para o desenvolvimento e para a modificação da cognição (Figueira, 1994).

Os objetivos cognitivos, implícitos ou explícitos, dizem respeito às metas que devem ser alcançadas e, por isso, promovem o progresso da atividade cognitiva do indivíduo. Eles representam um componente importante na monitorização, porque pelo conhecimento dos objetivos cognitivos o indivíduo orienta sua ação (Flavell, 1979).

As ações cognitivas correspondem às estratégias utilizadas para tornar mais eficaz o progresso cognitivo e avaliá-lo, e estão ligadas às realizações propriamente ditas. Ribeiro (2003) classifica as ações cognitivas em dois tipos: estratégias metacognitivas e estratégias cognitivas. Se as ações cognitivas estiverem voltadas ao progresso da monitoração centrada na avaliação da situação, elas podem ser entendidas como estratégias metacognitivas, as quais produzirão experiências metacognitivas e resultados cognitivos. Por outro lado, se forem utilizadas para produzir progresso cognitivo, em que a finalidade consiste em atingir o objetivo cognitivo, são entendidas como estratégias cognitivas, as quais produzem igualmente experiências metacognitivas e resultados cognitivos (Ribeiro, 2003).

Miller (1993) indica que uma das grandes mudanças no desenvolvimento, durante os anos escolares e na adolescência é a aprendizagem de como maximizar o uso das capacidades cognitivas mediante as capacidades metacognitivas. Por exemplo, no estudo acadêmico, à medida que adquirem maior experiência os alunos podem fazer melhor uso do tempo de estudo, selecionando os tópicos relevantes e ignorando os irrelevantes. Brown (1978) também observou que, com o passar do tempo, os estudantes aumentam consideravelmente o controle das estratégias que utilizam e de outros processos cognitivos. O estudo de Miller (1993) é apenas um exemplo de como tem sido observada a contri- buição da metacognição para a potencialização da aprendizagem. Neste sentido, os treinos que contemplam, além de atividades cognitivas, atividades metacognitivas, têm originado melhores resultados em termos de realização escolar. Segundo Paris e Winograd (1990), a metacognição, no que diz respeito especificamente à aprendizagem, pode assumir dois significados: a avaliação de recursos e a metacognição em ação. A avaliação de recursos ou autoapreciação cognitiva refere-se a reflexões pessoais sobre o estado dos conhecimentos e competências cognitivas, sobre as características da tarefa que influenciam a dificuldade cognitiva e sobre as estratégias disponíveis para a realização da tarefa. A metacognição em ação ou autocontrole cognitivo diz respeito a reflexões pessoais sobre a organização e planificação da ação - antes do início da tarefa - dos ajustamentos que se fazem enquanto se realiza a tarefa e nas revisões necessárias à verificação dos resultados obtidos (Ribeiro, 2003).

Deste modo, a eficácia da aprendizagem não é dependente apenas da idade, da experiência e do nível intelectual, mas também da aquisição de estratégias cognitivas e metacognitivas que possibilitem ao aluno planejar e monitorar o seu desempenho escolar; isto é, que lhe permitam a tomada de consciência dos processos que utiliza para aprender e a tomada de decisões apropriadas sobre as estratégias que utilizará em cada tarefa e, ainda, avaliar a sua eficácia, alterando-as quando não produzem os resultados desejados (Silva, \& Sá, 1993).

\section{Autoestima: possíveis relações com a metacognição, conceitos principais e impacto nos estudantes}

A metacognição envolve planejamento, monitoramento e regulação não só dos processos cognitivos, mas também dos afetivos e comportamentais envolvidos na aprendizagem (Boruchovitch, \& Santos, 2006). Cabe lembrar que o conceito de metacognição inclui as chamadas experiências metacognitivas, as quais, como já mencionado neste texto, exercem grande influência sobre a atividade cognitiva, uma vez que estão associadas à percepção do sucesso. Tais experiências também são entendidas como impressões de natureza cognitiva ou afetiva, de curta ou longa duração, que podem ocorrer antes, durante ou após a realização de uma tarefa (Boruchovitch, Schelini, \& Santos, 2010). A nova menção à metacognição precisou ser feita para que os aspectos afetivos não sejam entendidos como desvinculados dos processos metacognitivos.

No que se refere à autoestima, Coopersmith (1967), um de seus principais autores, considera que uma pessoa com autoestima alta mantém uma imagem bastante constante das suas capacidades, tem maior probabilidade de assumir papéis ativos em grupos sociais e tende a se orientar mais diretiva e realisticamente às suas metas pessoais. Assim, podemos inferir que um conhecimento ideal de nós mesmos e, consequentemente, um desempenho superior 
em tarefas envolvendo habilidades cognitivas/intelectuais, se dão quando essas duas capacidades, a de pensar sobre o próprio pensar e a de manter uma autoimagem positiva, são mantidas/desenvolvidas em conjunto.

A autoestima é o conjunto de atitudes que cada um tem sobre si mesmo, uma percepção avaliativa sobre si próprio e uma maneira de ser segundo a qual a própria pessoa tem ideias sobre si mesma, as quais podem ser positivas ou negativas. Não é estática, pois têm altos e baixos, revelando-se nos acontecimentos sociais, emocionais e psíquico-fisiológicos (psicossomáticos), emitindo sinais detectáveis em diversos graus. Ninguém deixa de pensar em si mesmo, temos a tendência de nos avaliar, no entanto o fazemos de um modo diferente, distinto, cada um à sua maneira, levando em conta o mundo ao nosso redor (García Del Cura, 2001).

A autoimagem é uma espécie de organização da própria pessoa, é composta de uma parte mais real e de outra mais subjetiva, convertendo-se em uma forma determinante e de grande significado para poder entender o meio ambiente em que se vive, tentando perceber significados antes atribuídos ao meio que depois ela vê que são seus (Mosquera, \& Stobaus, 2006). A autoestima, por sua vez, pode ser entendida como a avaliação que o indivíduo faz em relação a si mesmo, expressando uma atitude de aprovação ou desaprovação e indicando, como consequência, o grau em que ele se considera capaz, importante e valioso. Em suma, é um juízo de valor que o indivíduo mantém em relação a si mesmo (Coopersmith, 1967).

Abordar cientificamente o tema autoestima pode não parecer justificável, pois gera a sensação de que, por ser tão popularizada por livros de autoajuda, pelo senso comum e por ter se tornado uma palavra fácil na Psicologia das Relações Humanas, não faz sentido tal empreitada. Por outro lado, como sugere Mruck (1998), podem-se citar pelo menos cinco razões para justificar a necessidade de um enfoque científico para estudo da autoestima: 1- é um construto muito mais complexo do que pode parecer, pois está fortemente associado com outros aspectos da personalidade; 2- está relacionado à saúde mental ou bem-estar psicológico; 3- a sua carência se relaciona com certos fenômenos mentais negativos, como depressão e suicídio. Muitas pessoas que solicitam ajuda psicológica expressam com frequência sentimentos de inadequação, pouco valor e ansiedade associada à baixa autoestima; 4- é um conceito relevante para as ciências sociais; 5- é elevada a relevância social obtida atualmente.

Para Schunk (1990), os alunos que se sentem seguros das suas capacidades de aprendizagem e possuem um sentimento geral de competência, exibem comportamentos de interesse e motivação para as tarefas escolares. Este comportamento permite-lhes obter um bom desempenho acadêmico, fato que contribui para validar o seu sentimento pessoal de competência acadêmica e manter valores elevados de autoestima. Por outro lado, um aluno com resultados escolares negativos tenderia a sofrer o processo inverso, a menos que pudesse mobilizar formas de desvalorização desta informação negativa associada ao resultado escolar, para manter a autoestima em valores estáveis e aceitáveis.
A adolescência é marcada por inúmeras transformações orgânicas e psicológicas, as quais são profundamente influenciadas pela autoestima. O jovem busca a todo o momento a aprovação tanto do meio familiar quanto do grupo a que pertence. Nesta fase é extremamente importante refletir sobre a autoestima dos jovens, verificar seus pensamentos e atitudes no dia a dia, se são positivos ou negativos, pois a autoestima tem influência direta sobre o desempenho escolar.

Segundo Byrne (1996), o sucesso escolar apresenta-se como uma contingência importante na vida dos adolescentes, podendo influenciar a sua autoestima, embora o rendimento escolar esteja mais frequentemente associado à percepção de competência escolar ou autoconceito acadêmico; logo, a percepção de ser bom aluno ou de não ter dificuldades de aprendizagem está positivamente associada com a autoestima.

O vestibular ainda tem sido, na maioria das universidades brasileiras, a única forma de ingresso em um curso superior. Esse exame causa ansiedade, estresse e, muitas vezes, depressão no ano que precede a sua realização. Há a estimativa de que $92 \%$ dos alunos enfrentam problemas de estresse cognitivo e somático na elaboração e organização dos estudos para as provas de vestibular. Na maioria das vezes, estudar para o vestibular pode chegar a tornar-se algo patológico, ou seja, muitos jovens, durante o ano anterior ao exame, deixam de divertir-se e fazer outras coisas de que gostam, somente para estudar. O resultado disso é o aumento da ansiedade, pois os adolescentes acabam sentindo-se divididos entre a vontade de aproveitar situações diversas que lhes dão prazer e a obrigação de estudar, o que pode prejudicar a vontade de estudar e aprender o que estudam (Soares, \& Martins, 2010).

No último ano do Ensino Médio, para a obtenção do sucesso no vestibular, é necessário que o jovem, além de se preparar tecnicamente, tenha conhecimento de sua subjetividade e de suas capacidades cognitivas, para então monitorar tais aspectos e modificar estratégias de ação diante da necessidade de resolver problemas. A dinâmica que acontece no entrelaçamento dos aspectos cognitivos e metacognitivos e dos relativos à autoestima, dentro do contexto da adolescência, da escolha de uma profissão e do vestibular, faz com que a possível relação entre eles seja particularmente importante de ser pesquisada. Assim, o presente estudo pretendeu investigar a relação entre autoestima, nível intelectual geral e metacognição em estudantes do terceiro ano do Ensino Médio.

\section{Método}

\section{Participantes}

Foram escolhidas, de acordo com a disponibilidade, duas turmas do terceiro colegial de uma instituição privada de ensino, localizada em uma cidade do Interior do Estado 
de São Paulo. Os participantes foram 61 estudantes, inseridos nessas turmas, de ambos os sexos e com idade variando entre 16 e 18 anos.

\section{Materiais}

Para avaliar o nível intelectual geral dos participantes, foi utilizado o teste Matrizes Progressivas de Raven - Escala Geral (Oliveira, 2002), que é uma medida não verbal de inteligência. $O$ teste constitui-se de figuras inacabadas que devem ser completadas com uma das alternativas oferecidas. É composto por 60 itens e sua aplicação, no presente estudo, foi coletiva.

O material utilizado para avaliar o conhecimento e o controle metacognitivos foi uma adaptação, em que palavras foram modificadas a fim de tornar o instrumento mais próximo da realidade da faixa etária dos participantes, da Escala de Metacognição ([EMETA], Pascualon, \& Schelini, 2010), que é do tipo Likert de seis pontos, sendo que o participante, a partir da leitura de afirmações, tinha de escolher (assinalar) uma dentre seis possibilidades de resposta. As possibilidades de resposta eram representadas ao mesmo tempo por números (entre 1 e 6 ) e círculos, que iam aumentando de acordo com a numeração. Assim, se um participante considerasse que determinado item/afirmação da escala não era capaz de descrevê-lo ou representá-lo, ele deveria assinalar a primeira coluna com o número 1 acompanhado pelo menor círculo. Se, ao contrário, acreditasse que o item era capaz de representá-lo completamente, assinalaria a última coluna com o número seis acompanhado do maior círculo.

Em relação à autoestima, sua avaliação se deu por meio da escala de autoestima de Rosenberg - EAR. A EAR é uma escala de autorrelato em que o participante é apresentado a dez itens-afirmação sobre sentimentos de satisfação pessoal e deve escolher (assinalar) dentre as quatro opções decrescentes, aquela mais adequada à sua vida. A escala de autoestima de Rosenberg foi traduzida para o português e adequada aos contextos cultural e socioeconômico da população brasileira, bem como sua reprodutibilidade e validade foram devidamente demonstradas (Dini, Quaresma, \& Ferreira, 2004).

\section{Procedimento}

Foi contatada uma instituição particular de ensino, localizada em uma cidade do Interior do Estado de São Paulo. O contato foi feito pessoalmente e a pesquisadora explicou os objetivos, justificativa e procedimentos do estudo, bem como esteve à disposição para qualquer esclarecimento com relação à sua aplicação. Foram escolhidas, duas turmas de terceiro colegial, cujos alunos participaram dos procedimentos. Aos responsáveis por esses alunos foi enviada uma via do Termo de Consentimento Livre e Esclarecido, com cuja assinatura eles estariam concordando com que o aluno participasse do estudo.. O dia e o horário da aplicação do procedimento foram ajustados de acordo com a disponibilidade da instituição e a agenda de coleta do pesquisador.

A aplicação dos instrumentos foi coletiva, realizada em um único dia, com duração de 70 minutos, e se deu na seguinte ordem: primeiramente foi aplicado o teste Raven, logo depois a escala de metacognição e, por fim, a escala de autoestima. Após o término da análise dos resultados, os participantes e a instituição receberam uma devolutiva dos achados do estudo.

\section{Resultados}

Os resultados dos desempenhos nos testes psicométricos mostram que não houve relação entre nível intelectual geral e metacognição ou entre nível intelectual geral e autoestima, apenas evidenciando a presença de uma correlação positiva forte entre autoestima e metacognição. Serão descritos, inicialmente, os dados acerca do desempenho dos participantes nos três instrumentos aplicados para, posteriormente, serem apresentadas as correlações entre os escores das medidas.

A tabela 1 apresenta as médias e desvios-padrão obtidos pelos 61 participantes nos três instrumentos utilizados.

Tabela 1. Médias e desvios-padrão dos testes Raven, EMETA e Escala de Autoestima.

\begin{tabular}{lccc}
\hline & Média & Desvio-padrão & $\mathrm{N}$ \\
\hline Raven & 49,16 & 9,81 & 61 \\
EMETA & 166,57 & 17,50 & 61 \\
Escala de & & & \\
Autoestima & 30,27 & 4,55 & 61 \\
\hline
\end{tabular}

A tabela 1 indica que nas Matrizes Progressivas de Raven, a média de pontos foi 49,16 (D.P.= 9,81). O escore máximo possível (60 pontos) foi alcançado por dois participantes, e o menor escore (três pontos) foi obtido por apenas um dentre os 61 sujeitos do estudo. Em relação à Escala de Metacognição (EMETA), a média foi 166, 57 (D.P. = 17,50), sendo que dois participantes conquistaram a maior pontuação (203 pontos) e um obteve a menor delas (129 pontos), mas nenhum dos indivíduos colecionou o máximo possível de pontos (240 pontos). Na Escala de Autoestima de Rosenberg (EAR), a média de pontos foi 30,27 (D.P. $=4,55$ ). O escore máximo nesse teste (39 pontos) foi alcançado por dois participantes, enquanto um único sujeito obteve o escore mínimo (20 pontos), e novamente, nenhum sujeito conquistou a pontuação máxima possível (40 pontos). 
Tabela 2. Correlações de Pearson entre os escores dos testes Raven, EMETA e Escala de Autoestima.

\begin{tabular}{llll}
\hline & Escore total - Raven & EMETA & $\begin{array}{l}\text { Escala de } \\
\text { Autoestima }\end{array}$ \\
\hline Escore total - Raven & 1 & 0,097 & 0,037 \\
EMETA & 0,097 & 1 & $0,575\left(^{* *}\right)$ \\
Escala de Autoestima & 0,037 & $0,575\left(^{* *}\right)$ & 1 \\
\hline
\end{tabular}

** Correlação significativa a 0,01

A tabela 2 traz os dados de maior relevância para o presente estudo: os relativos à análise das correlações entre os três instrumentos aplicados.

A partir da obtenção dos coeficientes de correlação de Pearson, apresentados na tabela 2, pode-se dizer que houve uma correlação forte $(0,575)$ e significativa entre os escores da Escala de Metacognição (EMETA) e os da Escala de Autoestima (EAR), o que vai ao encontro do objetivo do estudo e aponta uma possível relação entre as variáveis conhecimento metacognitivo e autoestima; já as correlações de tais variáveis com o nível intelectual geral obtidas pelo Raven, foram fracas $(0,097$ para a EMETA e 0,037 para a EAR) e não significativas.

\section{Discussão}

Quando aspectos cognitivos, metacognitivos e relativos à autoestima são considerados dentro do contexto da adolescência, da escolha de uma profissão e do vestibular, ou seja, de um contexto típico do último ano do Ensino Médio, a possível relação entre tais aspectos pode ser um objeto de interesse da pesquisa psicológica. Dessa forma, o presente estudo pretendeu investigar a relação entre autoestima, o nível intelectual geral e a metacognição em estudantes do terceiro ano do Ensino Médio.

Para tal investigação, foram utilizados três diferentes instrumentos psicológicos - Matrizes Progressivas de Raven, Escala de Metacognição (EMETA) e Escala de Autoestima de Rosemberg (EAR) -, os quais avaliam a inteligência geral, a metacognição e a autoestima, respectivamente. A análise dos dados indicou uma correlação forte e significativa entre as pontuações obtidas nas escalas EMETA e EAR.

Quanto à correlação entre o nível intelectual geral, avaliado pelas Matrizes Progressivas de Raven, e a autoestima (avaliada pela EAR), os resultados obtidos no presente estudo indicam que os constructos nível intelectual geral e autoestima não apresentaram correlação forte ou significativa, ou seja, os maiores escores na tarefa destinada à avaliação do fator geral de inteligência não são acompanhados por escores maiores na medida de autoestima. Tais resultados parecem divergir dos achados de Byrne (1996), que afirma que o sucesso escolar é uma contingência impor- tante na vida dos adolescentes, podendo influenciar a sua autoestima. Além disso, o autor associa o bom rendimento escolar à percepção que o aluno tem de sua competência, percepção que neste estudo é representada pelo conceito de conhecimento metacognitivo, um dos componentes da metacognição avaliados pela EMETA. Novamente, os resultados encontrados apontam uma correlação fraca entre metacognição e nível intelectual geral.

Segundo Burochovitch e Santos (2006), a metacognição envolve planejamento, monitoramento e regulação não só dos processos cognitivos, mas também dos afetivos. Byme (1996) também indica que a percepção de ser bom aluno ou de não ter dificuldades de aprendizagem está positivamente associada com a autoestima, e neste caso, os dados obtidos vão ao encontro da literatura existente, do que é exemplo a correlação forte e significativa entre metacognição e autoestima.

Durante a fase de preparação para o vestibular, o adolescente enfrenta não só as incertezas relacionadas ao seu desempenho no dia da prova, mas também a forte cobrança da família e de amigos, situação que contribui para o surgimento da ansiedade e, em muitos casos, ultrapassa os limites da normalidade, prejudicando o desempenho do candidato. Muitas pessoas que prestam o vestibular diversas vezes podem não ser aprovadas por seu estado emocional e psicológico no dia da prova, o que é muito comum. O sentimento de obrigação de prestar vestibular e o fato de considerá-lo decisivo na vida são duas variáveis que têm efeito importante na ansiedade dos vestibulandos.

Por isso os resultados encontrados neste estudo, principalmente o dado referente à correlação entre conhecimento metacognitivo e autoestima, acrescentam informações importantes para a área da pesquisa relativa à relação entre os processos cognitivo e afetivo, bem como chama a atenção para as variáveis que implicam na alteração da qualidade do desempenho acadêmico dos jovens vestibulandos brasileiros.

\section{Considerações finais}

O presente estudo pretendeu investigar, através da aplicação de três testes psicométricos, a relação entre 
nível intelectual geral, a metacognição e a autoestima em estudantes do terceiro ano do Ensino Médio. Inteligência, metacognição e autoestima são aspectos essenciais do funcionamento cognitivo e afetivo. Esta pesquisa demonstrou que estes construtos não estão relacionados entre si, excetuando-se a metacognição e a autoestima, que tiveram seus escores, nos respectivos testes, correlacionados de modo forte e positivo. Confirma-se a suposição de Byme (1996) de que a percepção de ser bom aluno ou de não ter dificuldades de aprendizagem está positivamente associada com a autoestima. Ainda são necessárias mais pesquisas para investigar o papel do conhecimento metacognitivo associado a altos níveis de autoestima no sucesso escolar de estudantes brasileiros, principalmente daqueles que estão passando por avaliações para o ingresso na universidade. Uma maior investigação acerca da temática poderia significar o início do desenvolvimento de intervenções voltadas para o treinamento metacognitivo e para o aumento da autoestima de jovens estudantes, com vista a uma melhora do desempenho escolar e da qualidade de vida no período do vestibular.

\section{Referências}

Almeida, L. S. (1994). Inteligência: Definição e medida. Porto: Centro de Investigação, Difusão e Intervenção Educacional.

Anderson, M. (2004). Marrying intelligence and cognition: A developmental view. Em R.J. Sternberg, \& J. E. Pretz (Orgs.), Cognition and intelligence: Identifying the mechanisms of the mind. (pp. 268-287). New York: Cambridge University Pres.

Bernstein, J. (1961). Introdución: El test de Raven. Em J. C. Raven (Org.), Test de Matrices Progresivas: Escala general (pp. 13-46). Buenos Aires: Paidós.

Bolívar, C. R. (2002). Mediación de estratégias metacognitivas en tareas divergentes y transferencia reciproca. Investigación y Postgrado, 17 (2), 1-20.

Boruchovitch, E., \& Santos, A.A.A. (2006). Estratégias de aprendizagem: conceituação e avaliação. Em A.P.Noronha, A.A.A.Santos, \& F.F.Sisto (Orgs.), Facetas do Fazer em Avaliação Psicológica (pp. 107-124). São Paulo: Ed.Vetor.

Boruchovitch, E.; Schelini, P.W., \& Santos, A.A.A. (2010). Metacognição: Conceituação e medidas. Em A.A.A. Santos, F.F. Sisto, E. Boruchovitch, \& E. Nascimento (Orgs.). Perspectivas em avaliação psicológica (pp. 123-143). São Paulo: Casa do Psicólogo.

Brown, A. L. (1978). Knowing when, where, and how to remember: A problem of metacognition. Em R. Glaser (Org.), Advances in instructional psychology (pp. 367-406). New York: Halsted Press.

Relação entre autoestima... * * Luma Tiziotto Deffendi \& Patrícia Waltz Schelini
Brown, A. L. (1987). Metacognition, executive control, self-regulation, and other mysterious mechanisms. Em F.E.Weinert, \& R. H. Kluwe (Orgs.), Metacognition, Motivation, and Understanding (pp. 65116). Hillsdale, NJ: Lawrnce Erlbaum.

Byrne, B.M. (1996). Academic self-concept: Its structure, measurement, and relation to academic achievement. Em B.A. Bracken (Org.), Handbook of self-concept (pp. 287-316). New York: John Wiley \& Sons.

Coopersmith, S. (1967). The antecedents of self-esteem. San Francisco: Freeman.

Dini, g. M.; Quaresma, M. R., \& Ferreira, L. M. (2004). Adaptação cultural e validação da versão brasileira da Escala de Autoestima de Rosenberg. Revista da Sociedade Brasileira de Cirurgia Plástica, 19 (1), 41-52.

Dunslosky, J., \& Metcalfe, J. (2009). Metacognition. Los Angeles: Sage.

Faria, L.; Pepi, A., \& Alesi, M. (2004). Concepções pessoais de inteligência e auto-estima: Que diferenças entre estudantes portugueses e italianos? Análise Psicológica, 4 (22), 747-764.

Figueira, A.P.C. (1994). Inventário de Estratégias de Estudo e de Aprendizagem - Learning and Study Strategies Inventory - LASSI (Weinstein, \& Palmer, 1990) - Estudos de validação e adaptação. Psychologica, 12, 79-114.

Flavell, J. H. (1976). Metacognitive aspects of problem solving. Em L. B. Resnick (Org.), The nature of intelligence (pp. 231-235). Hillsdale, N.Y.: Erlbaum.

Flavell, J. H. (1979). Metacognition and cognitive monitoring: A new area of cognitive-developmental inquiry. American Psychologist, 34, 906-911.

García Del Cura, J. M. V. (2001). La autoestima en la escuela, la afectividad, actitudes y valores. Pamplona: Edición del autor.

Howard. R. W. (1993). On what intelligence is. British Journal of Psychology, 84, 27-37.

Mendoza, C. F., \& Colom, R. (2006). O estudo da inteligência no Brasil. Em C. Flores- Mendoza, \& R. Colom (Orgs.). Introdução à psicologia das diferenças individuais (pp. 189-200). Porto Alegre: Artmed.

Miller, P. (1993). Theories of Developmental Psychology. New York: W. H. Freeman.

Mosquera, J. J. M. \& Stobaus, C. D. (2006). Autoimagem, autoestima e autorrealização: qualidade de vida na universidade. Psicologia, Saúde \& Doenças, 7 (1), 83-88.

Mruck, C. (1998). Auto-estima: Investigación, teoría y práctica. Bilbao: Desclée de Brouwer. 
Oliveira, R. (2002). R1: teste não verbal de inteligência - Forma B. São Paulo: Vetor.

Paris, S. \& Winograd, P. (1990). How metacognition can promote academic learning and instruction. Em: B. Jones, \& L. Idol (Orgs.), Dimensions of thinking and cognitive instruction (pp.15-51). Hillsdale, N. J.: Erlbaum.

Pascualon, J. \& Schelini P. W. (2010). EMETA: Escala de Metacognição. Dissertação de Mestrado, Universidade Federal de São Carlos, São Carlos-SP.

Primi, R. (2003). Inteligência: avanços nos modelos teóricos e nos instrumentos de medida. Avaliação Psicológica, 1(2), 67-77.

Ribeiro, C. (2003). Metacognição: Um Apoio ao Processo de Aprendizagem. Psicologia: Reflexão e Crítica, 16 (1), 109-116.

Santos, A. A; Noronha, A. P. P., \& Sisto, F. F. (2005). Teste de Inteligência R1-Forma B e G36: evidência de validade convergente. Estudos de Psicologia, 10(2), 191-197.
Schunk, D. (1990). Self-concept and school achievement. Em C. Rofers, \& Kutnick (Orgs.), The social psychology of the primary school (pp. 70-79). London: Routledge.

Silva, A. L., \& Sá, I. (1993). Saber estudar e estudar para saber. Coleção Ciências da Educação. Porto: Porto.

Sisto, F. F.; Ferreira, A. \& Matos, M. P. B. (2006). TCR e R1: duas medidas do fator g. Revista de Psicologia da Vetor Editora, 7, (1), 69-77.

Spearman, C. (1927). The Abilities of Man. New York: Mc Millan.

Soares, A. B., \& Martins, J. S. R. (2010). Ansiedade dos estudantes diante da expectativa do exame vestibular. Paidéia, 20 (45), 57-62.

Sternberg, R.J. (1981). The evolution of theories of intelligence. Intelligence, 5, 209-230.

\section{Sobre as autoras}

Luma Tiziotto Deffendi (lumadeffendi@yahoo.com.br)

Graduada em Psicologia pela Universidade Federal de São Carlos e mestranda do Programa de Pós-Graduação de Psicologia pela mesma.

Patrícia Waltz Schelini (pws@ufscar.br)

Professora Adjunta dos cursos de graduação e pós-graduação em Psicologia da Universidade Federal de São Carlos 